\title{
Critical analysis of different current decomposition and compensation schemes
}

\author{
Jacques L. Willems, Fellow IEEE \\ Engineering Faculty, Ghent University, B-9052 Ghent, Belgium \\ Email: Jacques.Willems@UGent.be
}

\begin{abstract}
This paper discusses the fundamentals of different methods for current decomposition and non-active power compensation in polyphase power systems. The basic concepts and the results of the instantaneous active and reactive power theory on the one side, and of the active/reactive average power theory on the other side, are critically compared.
\end{abstract}

\section{INTRODUCTION}

Various algorithms have been developed for the generalization of the classical active/reactive power concepts, which are standard for single-phase sinusoidal power transmission, to polyphase nonsinusoidal voltages and currents. Two main lines of thought are

- the analysis based on average power properties, similar to the classical active/reactive power theory for single-phase sinusoidal systems,

- the anaysis based on properties of instantaneous voltages, currents and power.

A number of issues have been heavily debated, such as the questions below:

- are both approaches valid?

- which approach is the best?

- are both approaches suitable for compensation?

- are there situations where the former or the latter approach should be preferred?

In the present paper the basic ideas of both approaches are reviewed. The basic concepts are compared and the results are discussed. In particular it is shown that both lines of thought lead to valid power theories, but it depends on the situation at hand to see which approach is more appropriate.

\section{THREE FUNDAMENTAL APPROACHES TO CURRENT DECOMPOSITION}

The fundamental idea of current decomposition with the objective of compensation is to decompose the load current into components which are to be delivered by the source (the power system) and components which can be generated and delivered by a compensator at the load itself. The objective of the compensator is to generate the latter components of the current such that the source only has to deliver the former components. The decomposition is to be designed to obtain

- a better performance of the power or energy transmission from the source to the load (such as less transmission losses or less power fluctuations),
- a compensation current which satisfies the constraints corresponding to the compensation equipment used.

This immediately shows that the decomposition and the compensation techniques may be different depending on

- the definition of the performance of the power or energy transmission,

- the constraints due to the compensation equipment.

The energy required by the load should be delivered by the source; the compensator is assumed to generate no energy. The compensator may however store energy which is going back and forth between the compensator and the load. Another aspect is whether the compensator is a passive element (inductor or capacitor) or an active (electronic) element. The performance of the electrical energy transfer is in most studies characterized by the transmission losses; the expression of the losses may depend on the (relative) values of the resistances of the conductors of the transmission line. Usually it may be assumed that the phases of a multiphase line have the same resistance, which may however be different from the resistance of the neutral conductor. The efficiency of the electrical energy transfer may also be characterized by the absence of a current in the neutral conductor or by the presence of harmonics in the source current. Another aspect may be the level of the fluctuations in the power transmitted from the power system to the load.

The above considerations on the compensation and transmission efficiency lead to three fundamentally different concepts of current decomposition which are reviewed below. In the present section it is assumed that the transmission of energy is realized my a $m$-wire line; this may as well represent a line with $m$ phases without neutral conductor as a line with $m-1$ phases and a neutral conductor. To avoid unnecessary complexity it is assumed that all conductors have equal resistances. For the analysis of the cases where the resistances are unequal, the reader is referred to the literature [18]. Throughout this paper the currents and the voltages are assumed to be periodical with period $T$.

\section{A. Active and reactive current}

In the classical case of single-phase sinusoidal power transmission the decomposition of the current into active current and reactive current is well known. The active current is the smallest $^{1}$ current corresponding to the average or active power

\footnotetext{
${ }^{1}$ in the mean square sense
} 
and hence realizes the smallest energy losses in the supply line for the given energy transfer. The active current is also the current which corresponds to unity power factor. For the transmission of the active current the apparent power and the active power are equal.

A logical generalization of the concept of active current to the polyphase non-sinusoidal periodic case is obtained by defining it as the current vector which transfers the given active power for the given line voltages with the smallest r.m.s. current or equivalently with the smallest line losses. The result $^{2}$ is well known. The active current is given by

$$
\mathbf{i}_{a}(t)=\frac{P}{\left\|\mathbf{v}_{m o}\right\|_{r m s}^{2}} \mathbf{v}_{m o}(t)
$$

where the symbols and notations used are given in Appendix A. This expression shows that the active current vector is proportional to the voltage vector provided the voltages are referred to the voltage of the average voltage of the $m$ wires $^{3}$

The reactive current is the complement:

$$
\mathbf{i}_{r}(t)=\mathbf{i}_{m}(t)-\mathbf{i}_{a}(t)
$$

The given active power is delivered with minimal line losses if the source (power system) delivers the active current and the compensation equipment delivers the reactive current at the load terminals. The reactive current corresponds to zero active or average power, and hence zero energy transfer, but not to zero instantaneous power. The compensation equipment delivers no average power, but delivers or takes instantaneous power. It must be able to store electrical energy, as is the case for a capacitor or an inductor.

\section{B. Instantaneous power and powerless current}

In the technique introduced by Akagi and coauthors [1], [2], [3], it is assumed that compensation is performed by an element without energy storage, such as a power electronic converter. Then the constraint is that the instantaneous power delivered or consumed by the compensator is zero, such that compensation cannot change the instantaneous power (and hence obviously also not the energy) delivered by the source. Then the (instantaneous) power current ${ }^{4}$ is the current which delivers the instantaneous power with minimal instantaneous line loss. It is readily obtained as ${ }^{5}$ :

$$
\mathbf{i}_{p}(t)=\frac{p(t)}{\left\|\mathbf{v}_{m o}(t)\right\|^{2}} \mathbf{v}_{m o}(t)
$$

\footnotetext{
${ }^{2}$ The expression of the active current, as well as the expressions of the current decompositions in the other cases discussed in this paper, can be derived from the solution of an optimization problem with constraints, e.g. by means of the Lagrange multiplier technique.

${ }^{3}$ For the computation of the power the voltage reference is arbitrary. However for the computation of the active current a proper choice of the reference point for the voltages is essential [18].

${ }^{4}$ The instantaneous power current is also called the instantaneous active current.

${ }^{5}$ This expression requires that $\left\|\mathbf{v}_{m o}(t)\right\|$ is non-zero at all time $t$. For a realistic polyphase system this does not represent any loss of generality. However for a single-phase system this compensation technique, as well as the next one, is not applicable. Indeed, the voltage in a single-phase system vanishes at some times $t$, except for the unrealistic case of a large $\mathrm{d}$. c. component in the voltage.
}

The (instantaneous) powerless current ${ }^{6}$ is the complement:

$$
\mathbf{i}_{q}(t)=\mathbf{i}_{m}(t)-\mathbf{i}_{p}(t)
$$

The minimal line losses which can be realized by a compensator without energy storage are obtained if the source delivers the instantaneous power current and the compensator which corresponds to zero instantaneous power, delivers the instantaneous powerless current.

It is clear that the line losses are larger in the case of compensation without energy storage than in the case of compensation of the reactive current, because of the stricter constraints on the compensating equipment.

\section{Constant instantaneous power current}

For some applications it is interesting to achieve a constant (i. e. time-invariant) power delivered by the source (as in the case of balanced sinusoidal polyphase currents and voltages). Obviously to have the same energy transfer, this constant power must be equal to the average or active power. This is the fundamental idea of the technique which in some papers is called the modified Akagi technique. It leads to the decomposition technique with the constant instantaneous power current

$$
\mathbf{i}_{c}(t)=\frac{P}{\left\|\mathbf{v}_{m o}(t)\right\|^{2}} \mathbf{v}_{m o}(t)
$$

This is the smallest current (or the current with the smallest supply line loss) corresponding to the constant power $P$. The complement

$$
\mathbf{i}_{f}(t)=\mathbf{i}_{m}(t)-\mathbf{i}_{c}(t)
$$

can be associated with the power fluctuations. To achieve constant power transfer the compensation equipment should deliver the current $\mathbf{i}_{f}(t)$ and the source the current $\mathbf{i}_{c}(t)$. It is clear that the compensation does not deliver or consume energy, but should be able to store energy, since it should be able to modify the instantaneous power delivered by the source to the load. The losses are larger than in the case of compensation of the reactive current, as in II-A, because of the constraint of constant power and not only a constraint on the average power.

\section{DECOMPOSITION WITH ZERO NEUTRAL CURRENT}

In practice for systems with $m-1$ phases and a neutral conductor, it is often desirable to require zero neutral current [13], [14]. For the three cases considered in Section II the decomposition technique can be modified to obtain the smallest active current, the smallest instantaneous power current or the smallest constant instantaneous power current under the additional constraint of zero neutral current. The corresponding decompositions are readily obtained by solving the corresponding optimization problems. It turns out that the expressions of the currents in the $m-1$ phases are more or less the same as in the previous section. The only difference

\footnotetext{
${ }^{6}$ The instantaneous powerless current is also called the instantaneous reactive (or non-active, or deactive) current.
} 
is that the voltages should be referred to the average of the voltages of the $m-1$ terminals excluding the neutral terminal. Explicitly the currents are as given below for the $m-1$ phases. Since the sum of these currents vanishes, the source current at the neutral terminal is clearly zero. Below the expressions of the currents are given without explicit analysis, because of the complete similarity with the results in the previous section.

In each case the neutral line source current after compensation is zero, and the total load current in the neutral conductor is to be delivered by the compensator.

\section{A. Active and reactive current}

The active current in the $m-1$ phases is given by

$$
\mathbf{i}_{m-1, a}(t)=\frac{P}{\left\|\mathbf{v}_{m-1, o}\right\|_{r m s}^{2}} \mathbf{v}_{m-1, o}(t)
$$

The complement is the current to be delivered by the compensator for the $m-1$ phases:

$$
\mathbf{i}_{m-1, r}(t)=\mathbf{i}_{m-1}(t)-\mathbf{i}_{m-1, a}(t)
$$

\section{B. Instantaneous power and powerless current}

The instantaneous power current in the $m-1$ phases is given by

$$
\mathbf{i}_{m-1, p}(t)=\frac{p(t)}{\left\|\mathbf{v}_{m-1, o}(t)\right\|^{2}} \mathbf{v}_{m-1, o}(t)
$$

Here also the complement is the current to be delivered by the compensator for the $m-1$ phases:

$$
\mathbf{i}_{m-1, q}(t)=\mathbf{i}_{m-1}(t)-\mathbf{i}_{m-1, p}(t)
$$

\section{Constant instantaneous power current}

The constant instantaneous power current in the $m-1$ phases is given by

$$
\mathbf{i}_{m-1, c}(t)=\frac{P}{\left\|\mathbf{v}_{m-1, o}(t)\right\|^{2}} \mathbf{v}_{m-1, o}(t)
$$

Again the complement is the current to be delivered by the compensator for the $m-1$ phases:

$$
\mathbf{i}_{m-1, f}(t)=\mathbf{i}_{m-1}(t)-\mathbf{i}_{m-1, c}(t)
$$

\section{DISCUSSION}

The algorithm discussed in II-A corresponds to the early work of Fryze and has been extensively discussed and generalized in the work of Czarnecki. The techniques of II-B and II-C are usually attributed to Akagi and coworkers, although it should be emphasized that much of the ideas are already present in the early work of Depenbrock. Also much work on the interpretation and the generalization of the algorithms related to instantaneous power theory is presented in the papers by Ferrero and coworkers.

The three algorithms discussed in section II correspond to objective of delivering the average power required by the load by a modified source current by compensating the load current by delivering at the load terminals a suitable compensation current but no average power (or energy). Moreover the compensation strategy is designed such that within the constraints of the algorithm the transmission loss after compensation is as small as possible. In the algorithms II-A and II-B the decomposition leads to two orthogonal ${ }^{7}$ current components. This means that the transmission loss without compensation is equal to the sum of the loss with compensation and the loss corresponding to the compensating current.

This analysis leads to the following features:

- In the procedures of II-B and II-C the losses after compensation are larger than (or in particuarl cases equal to) the losses obtained by compensation according to the algorithm of II-A, since additional constraints are imposed.

- The transmisison losses are reduced by the compensation in the techniques of II-A and II-B except for special cases in which the losses are unchanged.

- It can not be said a priori whether the compensation technique of II-C leads to a reduction or an increase of the transmission losses.

Similar observations can be made for the procedures of Section III. It is clear that the losses by the algorithms of Section II are less (or equal in special cases) than by the corresponding algorithm of Section III. Indeed the constraint of obtaining zero neutral current reduces the potential performance of loss reduction. For this reason the losses after compensation may be larger than before compensation.

The procedures of Section III can be also related to the procedures of Section II in the following way: the compensation consists of forcing the zero sequence load current to be delivered completely by the compensator; the optimal source current is such that the total load power (including the zero sequence power) is delivered by the $m-1$ phases in the same way as in Section II, but with $m-1$ wires instead of $m$ wires. The compensator delivers zero total power, but delivers zero sequence power (if the load zero sequence power is nonzero), but this is compensated by the other powers in the compensator.

The techniques of II-A (and III-A) and II-C (and III-C) require a compensator with energy storage, the algorithm of II-B (and III-B) does not.

It is clear from the expressions in the previous section that in the techniques of II-B or II-C (and in III-B or III-C) harmonics which are not present in the voltage or not in the load current, may be introduced in the source current by compensation. This is not surprising since the compensation techniques only considers instantaneous behavior: the objective of minimal instantaneous lline losses and the constraint that the instantaneous source power is equal to either the instantaneous load power or the average load power. The introduction of harmonics can be avoided if this is imposed as an additional constraint. The reader is referred to [16] for an analysis in that sense. Note that the analysis of II-C (and of III-C) shows that in general there is an essential difference between reduction

\footnotetext{
${ }^{7}$ Note that for II-A orthogonality should be considered with respect to the scalar product integrated over the period, wheras for II-B orthogonality should be considered with respect to the usual scalar product of the instantaneous vectors.
} 
of harmonics in the instantaneous power and reduction of harmonics in the source current.

Further analysis is possible to identify the causes of the non-active current. For the technique of II-A the reader is referred to the work of Czarnecki who nicely decomposes the reactive current in various components respectively due to unbalanced load, to the frequency dependence of the resistive component of the load impedance (the scattering current), and to the inductive or capacitive character of the load.

Note that the procedure of II-B is a purely instantaneous technique; the compensation current is derived from instantaneous measurements of voltage and power. The technique of II-A only deals with average quantities; the active current is proportional to the load voltage with a proportionality factor which only depends on average power and average data on the voltage. The algorithm of II-C is hybrid, since the compensation itself is instantaneous (no memory in the compensator), but the required power after compensation is an average quantity; the proportionality between the desired source current and the voltage depends on instantaneous and average quantities.

The difference between the power current, defined in II-B, and the active current, defined in II-A, is called variation current by Depenbrock [9]. The reason is that according to his analysis this current causes the so-called non-intrinsic power oscillations. This may however be considered to be a confusing name. Indeed, as seen in II-C the oscillation of the intantaneous power can be made zero by a compensator with energy storage but without energy generation. Starting from the load current (or the uncompensated source current), it may be more logical to see it as follows:

- The instantaneous powerless current $\mathbf{i}_{q}(t)$ produces no power. The compensation of this current produces the instnataneous power current.

- The compensation of the difference between the instantaneous power current $\mathbf{i}_{p}(t)$ and the constant power current $\mathbf{i}_{c}(t)$ realizes zero variation (oscillation) of the instantaneous power. Would it not be logical to call this current the variation current and $p(t)-P$ the variation power?

It should be noted that the algorithm of II-A is concerned with the performance of the energy transfer, whereas the techniques of II-B and II-C concern the (instantaneous) power transfer.

Often in the literature a distinction is made between decompositions in the time domain and decompositions in the the frequency domain. It is the opinion of the author that there is no fundamental difference from that point of view. Some decompositions can be more conveniently analysed in the frequency domain, others in the domain domain. This is only a matter of convenience for the analysis. The basic ideas are however independent of the way the analysis is carried out. For example, the results of II-A are expressed and discussed easily in the frequency domain. In the present paper they are shown in the time domain because the comparison with the other algorithms is more transparent.
There are still numerous other algorithms which start from other objectives. The lead to a similar analysis and often to expressions which are comparable. For example one may wish that a compensator without energy generation (but with the possibility of energy storage) realizes a balanced positive sequence source current although the voltage is unbalanced and distorted. The optimal compensation (with minimal line losses) leads to the optimal source current (for the given active or average power):

$$
\mathbf{i}_{p s}(t)=\frac{P}{\left\|\mathbf{v}_{p s}\right\|_{r m s}^{2}} \mathbf{v}_{p s}(t)
$$

where $\mathbf{v}_{p s}(t)$ is the positive sequence voltage vector at fundamental frequency of the voltage at the load terminals. The current to be delivered by the compensator is hence

$$
\mathbf{i}_{\text {comp }}(t)=\mathbf{i}_{m}(t)-\mathbf{i}_{p s}(t)
$$

\section{COMMENTS ON MISINTERPRETATIONS AND MISUNDERSTANDINGS}

In the literature a number of objections against some of the algorithms discussed in this paper have been raised. It is my conviction that most of them are due to misconsception or to requiring some features which are not the objective of a particular technique.

It is the feeling of the author that one of the reasons why the instantaneous power theory is often not well understood is that the original papers by Akagi and coauthors give the impression that the use of the Clarke or Park transformation is fundamental for the algorithm. It is my conviction that the transformation should only be seen as a tool for the analysis, nothing more. The ideas can equally well be understood, even better, without using a transformation.

Moreover compensation is fundamentally a matter of currents and current decomposition. Indeed the main objective of compensation is to modify the source or power system current such that the power or energy required by the load is delivered in a better way, with better characteristics for the power system, with less losses, ... Nevertheless most formulations concetrate on power. Certainly in more complex cases it may be confusing to concentrate on power. Note that (ideal) compensation does not change either the instantaneous or the average power. Not power components, but current components are compensated to reduce the transmission loss. To state it even more bluntly, power decompistion is fictive, current decomposition is real.

Also in the literature often confusion exists between techniques II-B and II-C. The reason is probably that the term constant power is sometimes interpreted as constant with respect to compensation (technique II-B), and in other papers as constant in time (technique II-C).

Another reason of misconception is the following. In classical power theory in technique II-A, the concepts of active current and reactive current are obtained as the currents corresponding to active power and reactive power, or to the current corresponding to the minimum average losses and the redundant current. These currents are periodic currents, where 
the active current has the same waveform as the voltage. For these periodic currents the instantaneous values may be considered: the instantaneous value of the active current and the instantaneous value of the reactive current. On the other hand in instantaneous power theory, in particular in technique II-B, the instantaneous current is decomposed in a power carrying current component which is necessary for the instantaneous power transfer, and which my be called the instantaneous active current, and a current component which is not necessary, the powerless current component, which may be called the instantantaneous reactive current ${ }^{8}$. Note that the instantaneous active current is not the same as the instantaneous value of the active current. Similarly the instantaneous reactive current is not the same as the instantaneous value of the reactive current. Nevertheless their sums agree, namely the instantaneous current.

In particular L.S. Czarnecki (see e. g. [4], [7]) explicitly formulates a number of objections to instantaneous power theory. Below some of these objections are discussed with the purpose of showing that the facts may be correct, but that these objections do not invalidate the instantaneous power theory.

- Czarnecki [7] claims that the instantaneous power theory cannot be right since an instantaneous reactive current can occur in supply lines of purely resistive loads, for example because of the unbalance of a resistive load. This is not an error of instantaneous power theory since even in the case of a resistive, but unbalanced load the instantaneous power can be delivered by an instantaneous current which is not the smallest possible current, or otherwise stated, which does not correspond to the smallest possible losses for the given power transfer. Then the instantaneous current can be decomposed into a current component which is neccesary for the power transfer (and which is called instantaneous active current) and a component which is redundant and causes additional losses and may be compensated (and which is called instantaneous reactive current). Hence the existence of an instantaneous reactive current should not be associated to the classical idea of reactive elements, such as inductive and capacitive elements.

- In the same paper Czarnecki [7] points out that according to instantaneous power theory an instantaneous active current may exist in supply lines for a purely reactive load, where the reactive load is considered in the classical sense of average power theory. Again this is no surprise since even a reactive load (inductor or capacitor) instantaneously delivers or consumes power for energy storage, and hence this correponds to an instantaneous active (power carrying) current.

- A third objection formulated in that paper [7] is that distorted current components may be obtained in the decomposition of the current for a sinusoidal voltage and a linear load. This is indeed true and may certainly

\footnotetext{
${ }^{8}$ To avoid confusion with the classical notion of reactive elements, sometimes the terms non-active or deactive are used instead of reactive.
}

be considered as a drawback of compensation without energy storage. However it is not an erroneous conclusion of instantaneous power theory. If the objective is to deliver the instantaneous power with the smallest possible loss (compensation without energy storge), and this objective is realized at every time independently, then the compensation current is computed independently at every time instant. Hence no a priori properties of the global waveform will be guaranteed.

The conclusion on Czarnecki's objections is that they may be valid objections to the performance of compensation by means of the instantaneous power approach. In other words, does an objective of compensation without energy storage lead to acceptable results? Does the objective of compensation with the objective of constant instantaneous power lead to acceptable results? However these questions do not invalidate the theory of instantaneous power and the corresponding current decomposition algorithms. The theory of current and power decompositions, or the theory leading to the decomposition of current into the minimal current needed for the power transfer with the particular objectives and constraints, and its complement is a valid theory for the analysis of instantaneous power properties; if one only analyses the instantaneous power properties, it is obvious that no average properties are obtained. They yield however insight in other aspects of the power transfer between source and load.

\section{CONCLUSIONS}

In this paper the principles of different methods for current decomposition and non-active power compensation in polyphase power systems are discussed. The basic ideas and the results of the instantaneous reactive power theory on the one side, and of the active/reactive average power theory on the other side, are critically compared. It is shown that it is not a question of 'which method is right or wrong?', but that the relevant questions are 'what do want to achieve by compensation? which compensation elements are available? what information are we interested in?'.

The objective of this paper is to contribute to clarify some misinterpretations and misunderstandings connected to instantaneous power theory. Hopefully the paper will also help to show that the various power theories are not contradictory, but complementary.

\section{APPENDIX A NOTATIONS}

The following notations and symbols are used in this paper: The vector of the $m$ load currents is denoted by $\mathbf{i}_{m}$, and the $m$ components by $i_{1}, i_{2}, \cdots, i_{m}$.

The vector of the $m$ load voltages is denoted by $\mathbf{v}_{m}$, and the $m$ components by $v_{1}, v_{2}, \cdots, v_{m}$.

The vectors without the $m$-th component (current or voltage of the neutral terminal) are denoted by $\mathbf{i}_{m-1}$ and $\mathbf{v}_{m-1}$.

The (Eucildean) norm of a vector $\mathbf{v}$ with $n$ components is denoted by $\|\mathbf{v}\|$ and is equal to

$$
\|\mathbf{v}\|=\sqrt{v_{1}^{2}+v_{2}^{2}+\cdots+v_{n}^{2}}
$$


The rms value of a time-dependent periodic vector $\mathbf{v}(t)$ is

$$
\|\mathbf{v}(t)\|_{r m s}=\sqrt{\frac{1}{T} \int_{0}^{T}\|\mathbf{v}(t)\|^{2} d t}
$$

where $t$ denoted the time variable and $T$ the period.

The instantaneous power corresponding to the line current vector $\mathbf{i}_{m}$ and the voltage vector $\mathbf{v}_{m}$ is

$$
p(t)=\mathbf{v}_{m}(t)^{T} \mathbf{i}_{m}(t)
$$

where the voltages may be referred to an arbitrary reference point, under the assumption that the sum of the currents is zero. For periodic voltages and currents the average value of the power

$$
P=\frac{1}{T} \int_{0}^{T} p(t) d t
$$

is called the active power.

The average value of the $m$ components of the vector $\mathbf{v}_{m}$ is denoted by

$$
v_{o m}=\frac{1}{m}\left(v_{1}+v_{2}+\cdots+v_{m}\right)
$$

and the average of the components of $\mathbf{v}_{m-1}$ is

$$
v_{o, m-1}=\frac{1}{m-1}\left(v_{1}+v_{2}+\cdots+v_{m-1}\right)
$$

For some of the expressions in this paper the voltages should be measured with respect to a particular voltage reference. For that reason specific notations are used:

$\mathbf{v}_{m o}$ is the vector of the load voltages where the voltages are referred to $v_{o m}$.

$\mathbf{v}_{m-1, o}$ is the vector of the $m-1$ load voltages, omitting the $m$-th terminal corresponding to the neutral conductor, where the voltages are referred to $v_{o, m-1}$.

\section{ACKNOWLEDGMENT}

The author wishes to pay tribute to Prof. Manfred Depenbrock who in his early work in the sixties of the $20^{t h}$ century already obtained results which have been rediscovered and debated decades later.

\section{REFERENCES}

[1] H. Akagi, Y. Kamazawa and A. Nabae, "Instantaneous reactive power compensators comprising switching devices without energy storage components," IEEE Trans. Ind. Appl., vol. 20, pp. 625-630, 1984.

[2] H. Akagi, A. Nabae and S. Atoh, "Control strategy of active power filters using multiple voltage-source PWM converters," IEEE Trans. Ind. Appl., vol. 22 , pp. 460-465, 1986.

[3] H. Akagi and A. Nabae, "The $p-q$ theory in three-phase systems ounder non-sinusoidal conditions," Eur. Trans. Elect. Power, vol. 3, pp. 27-31, 1993.

[4] L.S. Czarnecki, "On some misinterpretations of the instantaneous reactive power theory," IEEE Trans. Power Electronics, vol. 19, pp. 828-836, 2004.

[5] L.S. Czarnecki, "Currents' Physical Components (CPC) in circuits with nonsinusoidal voltages and currents. Part 1: Single-phase linear circuits," EPQU Journal, vol. XI, No 2, pp. 27-48, 2005.

[6] L.S. Czarnecki, "Currents' Physical Components (CPC) in circuits with nonsinusoidal voltages and currents. Part 2: Three-phase linear circuits," EPQU Journal, vol. XII, No 1, pp. 3-13, 2006.

[7] L. S. Czarnecki, "Instantaneous reactive power p-q theory and power properties of three-phase systems," IEEE Trans. Power Delivery, vol. 21, pp. 362-367, 2006.

[8] M. Depenbrock, "The FDB-method, a generally applicable tool for analysing power relations," IEEE Trans. Power Systems, vol. 8, pp. 381386, 1993.

[9] M. Depenbrock, "Variation power, variation currents: physical background and compensation rules," Eur. Trans. Elect. Power, vol. 11, pp. 309-316, 2001.

[10] M. Depenbrock, V. Staudt and H. Wrede "A theoretical investigation of original and modified instantaneous power theory applied to four-wire systems," IEEE Trans. Ind. Appl., vol. 39, pp. 1160-1167, 2003.

[11] A. Ferrero, "Mathematical foundations of the instantaneous power concepts: an algebraic approach," Eur. Trans. Elect. Power, vol. 6, pp. 305-309, Sept/Oct 1996.

[12] A. Ferrero, "Definitions of electrical quantities commonly used in nonsinusoidal conditions," Eur. Trans. Elect. Power, vol. 8, pp. 235-240, 1998.

[13] P. Salmerón et al., "Compensation in nonsinusoidal unbalanced threephase four-wire systems with active power-line conditioner," IEEE Trans. Power Delivery, vol. 19, pp. 1968-1974, 2004.

[14] P. Salmerón and R. S. Herrera, "Distorted and unbalanced systems compensation within Instantaneous reactive power framework," IEEE Trans. Power Delivery,, vol. 21, pp. 1655-1662, 2006.

[15] J. L. Willems, "A new interpretation of the Akagi-Nabae power components for nonsinusoidal three-phase circuits," IEEE Trans. Instrum. Measurement, vol. 41, pp. 523-527, 1992.

[16] J. L. Willems and D. Aeyels, "New decomposition of 3-phase currents in power systems," IEEE Proc. C, vol. 140, pp. 307-310, 1993.

[17] J. L. Willems, "Critical analysis of the concepts of instantaneous power current and of active current," Eur. Trans. Elect. Power, vol. 8, pp. 271274, 1998.

[18] J. L. Willems, J. A. Ghijselen, and A. E. Emanuel, "The apparent power concept and the IEEE Standard 1459-2000," IEEE Trans. Power Delivery, vol. 20, pp. 876-884, 2005. 